\title{
La Base de Datos de Ciudades Históricas Andaluzas
}

\section{Carlos Tapia Martín}

Centro de Documentación del IAPH. Área de Patrimonio Arquitectónico y Urbano

Con claridad, el lugar donde debe presentarse este trabajo es el de "Tendencias". En la disquisición posible sobre la elaboración de un producto informático o el referir las circunstancias reflexivas, implementan en este caso una importante consideración que debe mencionarse para así posicionarnos argumentativamente. En realidad, la situación particular del IAPH, mejor, de su propia identidad, puede provocar inquietudes entre quienes persiguen fines semejantes desde distintas posturas. De una parte, la reglamentación y la gestión cultural, y no muy lejos, la investigación sobre los modelos de entendimiento patrimonial con sus distintas derivaciones. Alguna de ellas se concentra en fundamentar la eficacia de la gestión con el pragmatismo, y otras ramificaciones apuestan por ser en sí mismas extensiones de la investigación, puesto que sólo así creemos posible que el concepto cultural asuma un dinamismo le que permita legislarse y evitar el riesgo de lo contrario. Por ello, el trabajo que se relata aquí debe ser entendido como una intromisión en el campo de la experimentación, con objetivos que aúnan esas dos vertientes que se separan y se reúnen intermitentemente. Esos pretendidos alcances se van a estructurar en diferentes fases y "Ciudades Históricas Andaluzas" va a ser una de los primeros ensayos.

En cualquier caso, podemos decir que no existe el factor riesgo al plantear tesis como las que vamos a enunciar o, al menos, no existe en cuanto a discrepancias en los entendimientos o ámbitos de conocimiento y operatividad. Y es que la apertura de miras que se va obteniendo por los años de trabajo conscientes y atentos a cuanto podía ser fuente y aliento del hacer patrimonial, da frutos a veces jugosos. Nombrar el riesgo implica reconocer que existe, como también la equivocación, pero no será sino con la reflexión y con la investigación como se mantenga una postura actualizada y coherente. A pesar de ello, el contacto con otros proyectos e identidades culturales, permite afirmar que el conocimiento de lo que somos no vendrá dado por el desarrollo exhaustivo de la documentación de sus señas, sino por las relaciones entre ellas. Este planteamiento es una sensibilidad que se impone incluso en los marcos de gestión y da lugar a una estrategia bastante común hoy: planes subregionales o de índole superior, mancomunidades (que habla de saltos geográfi-

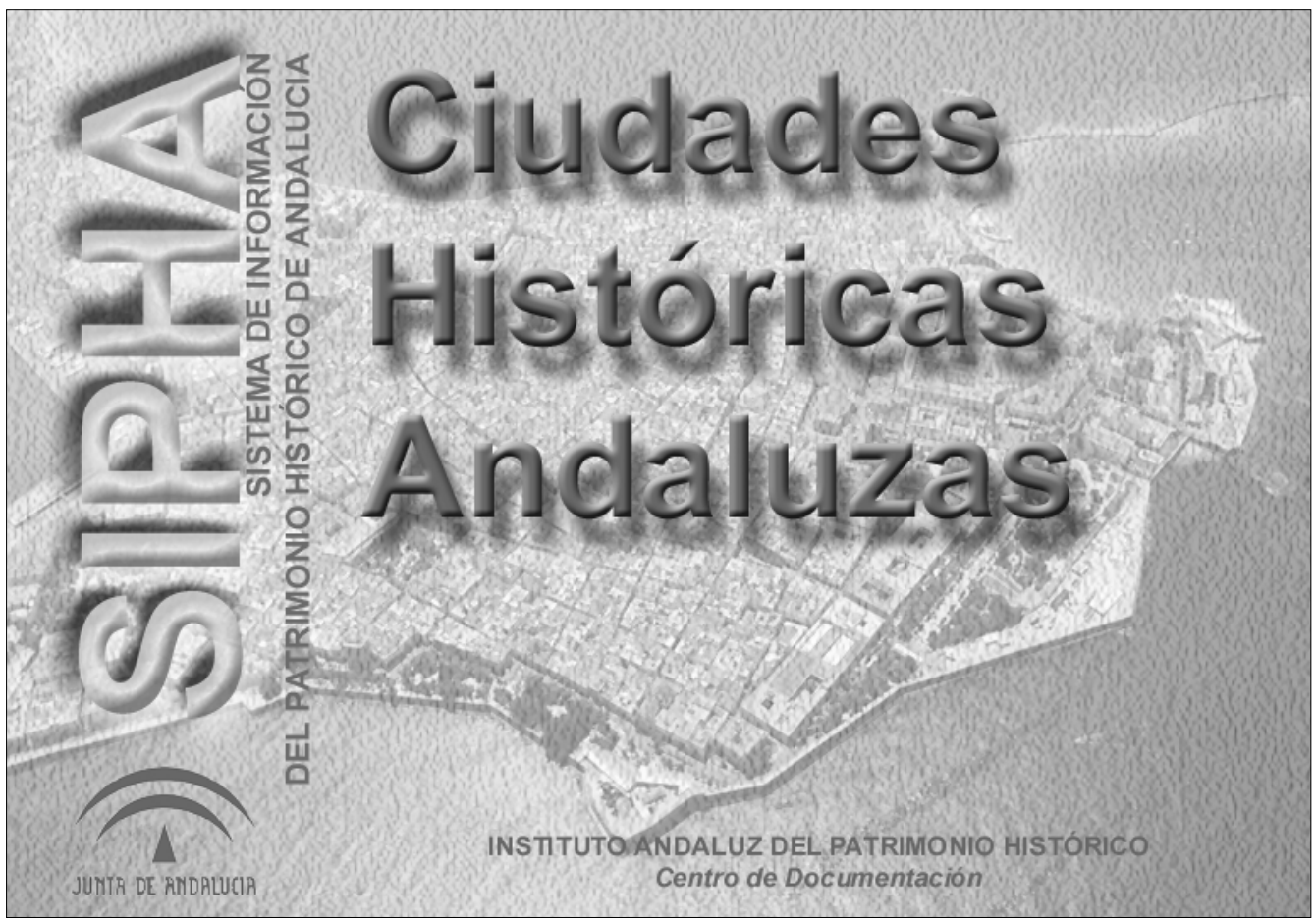

Imagen I. Máscara de entrada a la base de Ciudades Históricas 
cos no administrativos), áreas metropolitanas, comarcas, zonas de desarrollo o protección medioambiental. Un nombre define esta aproximación: el territorio.

Antecedentes pueden encontrarse en lo que respecta al IAPH en algunos ensayos producidos por el Área de Arqueología del Centro de Documentación, basados en modelos analíticos que se tradujeron en pruebas de bases de datos contrastadas con los resultados que se iban obteniendo en paralelo con instituciones que planificaban sus desarrollos, o la incorporación de herramientas de georreferenciación a las existentes. Al mismo tiempo, los mecanismos docentes relacionados o pertenecientes al IAPH ponían a disposición de sus centros de investigación el programa de sus enseñanzas. La acción combinada del Máster de Arquitectura y Patrimonio, que propone estudios de prototipos territoriales, recabando información y modos de producirla, con el Centro de Documentación, que trata de dotar de nuevos sentidos desde la definición de área territorial hasta la conjunción de formas de analizar y recabar la información necesaria para que ésta sea establecida, posibilitará a medio plazo un garante conciliador entre muchas perspectivas que trabajan en este campo. Este proyecto, iniciado con el estudio del Valle del Río Guadalquivir (eje Sevilla-Córdoba) habrá de seguir concretándose en sucesivas fechas y será objeto de otros escritos que irán viendo la luz a medida que se avance en sus pretensiones.

Otras circunstancias previas que ya han sido publicadas, pero que son las referencias necesarias para encuadrar el proyecto son la base de datos de "Conjuntos Históricos" o la propia renovación de la base de inmuebles "Sibia": la incorporación de elementos analíticos relacionales en Sibia ("estancias" en primera instancia y "unidades" ahora) y el trabajo de recopilación de información y las consideraciones establecidas en su momento para entender y proteger las áreas urbanas reconocidas como Conjuntos Históricos. Y así, la figura de Conjunto Histórico responde a la clasificación que la legislación patrimonial hace respecto a los inmuebles y lo define como "la agrupación de bienes inmuebles que forman una unidad de asentamiento, continua o dispersa, condicionada por una estructura física representativa de la evolución de una comunidad humana por ser testimonio de su cultura o constituir un valor de uso y disfrute para la colectividad. Asimismo es Conjunto Histórico cualquier núcleo individualizado de inmuebles comprendidos en una unidad superior de población que reúna esas mismas características y pueda ser claramente delimitado".

Pero, como sabemos, delimitar un área como de especial protección por sus determinadas características históricas, arquitectónicas y ambientales no puede obviar la realidad de su pertenencia a un núcleo urbano o a un ámbito territorial mucho más amplio. No son las similitudes, sino las conexiones históricas o interpretativas que desde el presente -y desde el análisisse deben hacer. No hay historia sino desde el presente, no es posible otra definición de historia. Los marcos comerciales entre ciudades o la presencia territorial de núcleos poblacionales, zonas de producción o
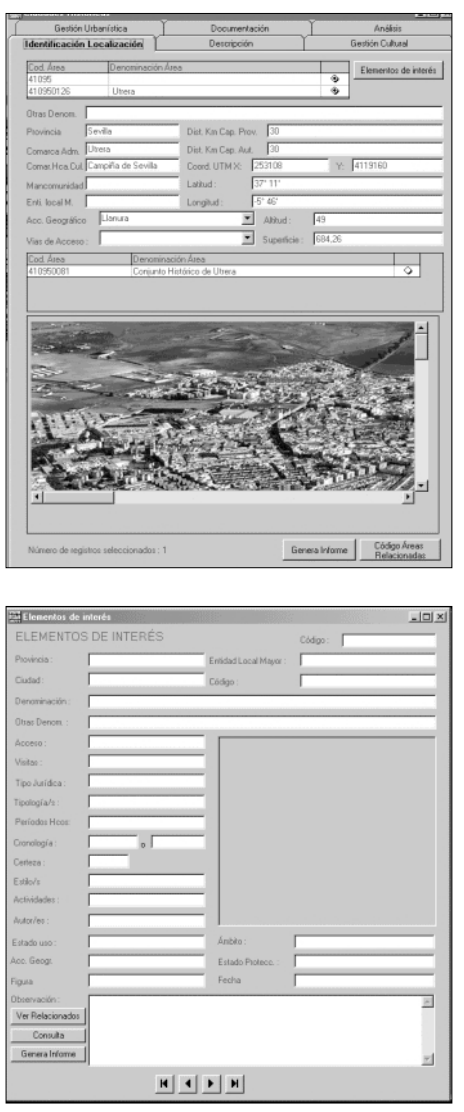

Imagen 2. Pantalla de datos identificativos y de localización

(2)

Imagen 3.

Pantalla de elementos de interés.

de enfrentamiento han decantado la existencia de los asentamientos desarrollados actuales, sus vías de comunicación, su mezcolanza sanguínea y por consiguiente cultural, que posiciona en una nueva comprensión los tradicionalmente elementos singulares representantes de la riqueza patrimonial local.

Por ello el Centro de Documentación del IAPH se ha planteado la ampliación de estos ámbitos protegidos mediante una nueva base de datos que extiende el objeto de estudio a todas las Ciudades Históricas de Andalucía y las somete a la observación cautelar de sus conocimientos relacionales territoriales.

La nueva base contempla esta ampliación como básica para el entendimiento de nuestros bienes patrimoniales intentando relacionarse con otras experiencias ya iniciadas en otras Consejerías en el ámbito territorial como el Sistema de Ciudades Andaluzas (Consejería de Obras Públicas y Transportes).

En primera fase, se centra el estudio en el ámbito urbano, siendo el inicio de prueba de lo que será la indagación territorial de Andalucía. Y se hace montando una aplicación informática de visualización de cartografía y la base de datos que reelabora la anterior de Conjuntos Históricos. Dicha aplicación tiene como objetivo el desarrollo y profundización de la información de las Ciudades Históricas Andaluzas que mediante la compatibilización de los GIS y las Bases de Datos del Patrimonio Histórico de Andalucía pone en relación la problemática de las ciudades en su mediación entre el ámbito territorial y el edificatorio. 

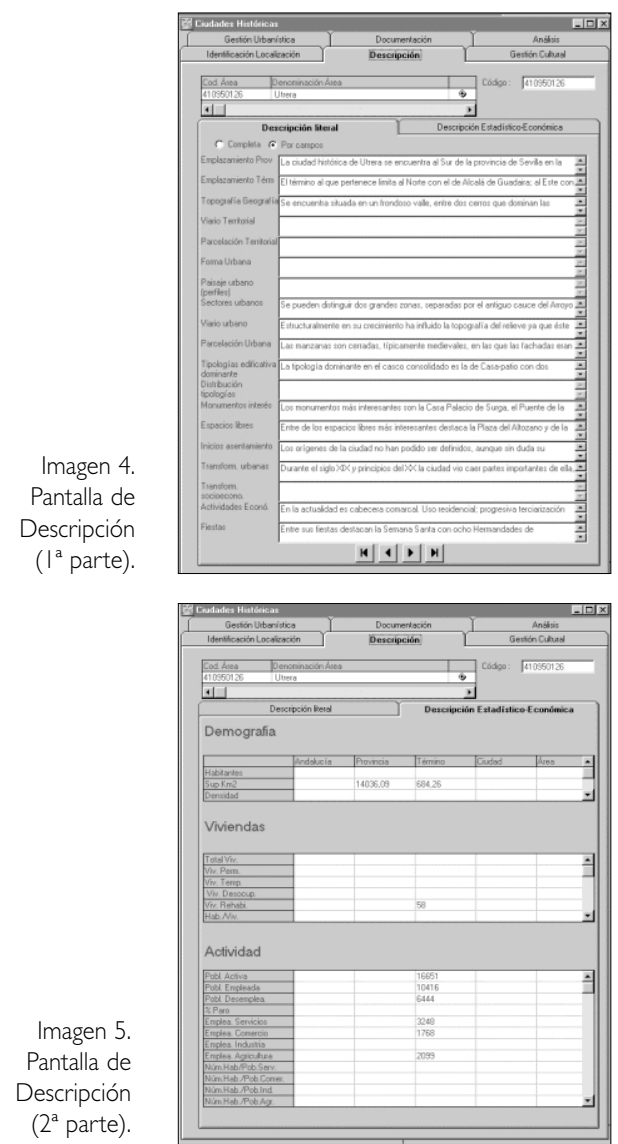

La aplicación se plantea con un objetivo ambicioso donde además de facilitar información de identificación, localización, descripción y documentación de nuestro patrimonio urbano, posibilita la gestión cultural y urbanística, y que pueda facilitar diversos análisis (físico-formal, funcional, económico, legal, medioambiental e histórico) de las diferentes redes que conforman nuestras ciudades.

Partiendo el prototipo informático desarrollado para arqueología, donde a la base de datos Arqueos se le une un visualizador de elementos georreferenciados, se evoluciona para llegar a los objetivos fijados para esta fase. La consulta de datos y su ubicación territorial (por ahora urbana), se realiza al mismo tiempo y bajo las relaciones establecidas por el analista que maneje el programa, ya que le requerirá que muestre las coberturas (datos georreferenciados) que necesite en cada caso. Recordar aquí que la Junta de Andalucía tiene en desarrollo y publicadas numerosas de estas coberturas que se actualizan periódicamente. El Instituto Cartográfico o las propias áreas del Centro de documentación manejan datos territoriales sobre yacimientos arqueológicos, inmuebles protegidos, inmuebles de interés etnológico, conjuntos históricos, perímetro de núcleos de población, orografía, hidrografía, recursos naturales y de producción, etc., lo que permite no tener que almacenar datos estáticos, sino asociarlos a su lugar y ser conjugados entre sí. Aunque la interacción entre estos "puntos inteligentes" todavía no es total, se pretende que en un futuro las bases funcionen con información dinámica completa- mente. Quiere decirse que si cada institución georreferencia su información, podría volcarse sobre el programa y servir al estudio en curso.

Éste es nuestro gran reto, conseguir normalizar toda la documentación que se está construyendo y poder volcarla sobre un visualizador común. Sin embargo, ese proceso de integración es la tarea más compleja, como lo demuestran los años que lleva el Centro de Documentación intentando dar forma a una base de datos que participe de estas reflexiones. Ya se dispone de una versión LT (limitada) y puede decirse que el planteamiento de las renovaciones de las bases sectoriales de patrimonio histórico nacen del requerimiento unificador. Incluso "Ciudades Históricas" y la futura base "Territorio de Andalucía" puede decirse que investiga desde una posición más avanzada estas cuestiones.

El momento es importante en el trabajo sobre la documentación. Las grandes dudas que se suscitan sólo se resuelven cuando se contrastan los principios y de hecho, al celebrarse los días 10, I I y 12 de Mayo la Euroconferencia sobre patrimonio y ciudad en el marco de los GIS (Sistemas de Información Georreferenciada) en Sevilla, se presentaron los avances obtenidos para así observar qué opiniones merecen a otros investigadores y qué aportaciones podemos obtener de la exposición de sus trabajos específicos.

Avanzamos ahora algunas de las pantallas más relevantes:

En primer lugar, continuando con las normalizaciones de bases de datos ya establecidas, abstraemos los campos documentales en conceptos identificativos y de localización, incluyendo aquí las 768 ciudades históricas andaluzas y las zonas en las que se compone. Una de ellas sería su Conjunto Histórico (si lo tuviera). De esta manera, se incide en el interés de mostrar cada elemento en su relación con otras circunstancias, y no sólo entre ciudades, sino dentro de cada una de ellas. Aparecen los campos que se creen más adecuados tanto en cada base sectorial (arquitectónica, etnológica, arqueológica...) como en la base integrada, de modo que los futuros volcados de información se harán con facilidad.

Siguiendo con el recorrido, se dispone un botón de acceso que lleva al tratamiento de aquellos elementos de interés que se refieran a la ciudad y a la zona especificada. Con ello se quiere mantener un criterio claro en cuanto a la nueva consideración que se quiere dar a las relaciones. No es el fin último dar información sobre el elemento singular, sino que dentro del marco relacional, es necesario que los elementos catalogados por cualquier instancia sean reconocidos y presentados. Las fuentes de esta pantalla serán todas las bases sectoriales, eligiendo los campos mínimos para su identificación.

Para continuar con la navegación analítica, nos detendremos ahora en la descripción de la ciudad. Para ello contamos con una serie de campos que pretenden que todos los registros sean estudiados bajo los mismos aspectos. Pero para una lectura comprensiva y continuada existe la posibilidad de pulsar un botón 
que presenta todos los datos en un solo campo descriptivo. Además, es una pantalla con una barra de desplazamiento que lleva a completar los argumentos descriptivos con datos socioeconómicos de ámbito autonómico, de mancomunidad, de comarca, de provincia, de ciudad, de su conjunto y de las subáreas en que queramos descomponer la ciudad. Se dispone siempre en un lateral de la pantalla, ya que se debe tener en cuenta la información gráfica que aparece en forma de planimetrías y de coberturas, que se manejan con las botoneras del menú superior y con los dos campos del menú inferior, posibilitando obtener cierta documentación asociada, como decíamos más arriba.

Dentro de los avances efectuados con este trabajo, encontramos tres pantallas destacables. La primera (imagen 6) es la gestión cultural, que se complementa con la gestión urbanística y el capítulo de análisis, donde se tratan como redes de información a las infraestructuras, los espacios edificados y las zonas libres.

Esta propuesta es una primera aproximación a los posibles análisis de las Ciudades Históricas Andaluzas. El planteamiento parte de dejar abierta la posibilidad del Área de Estudio que se estime necesario analizar. Así, se podrá ir desde el área más genérica como son las Ciudades Históricas pasando por los sectores del mismo, hasta la posibilidad de analizar algún subsector concreto. Esta manera de proceder también posibilita el análisis de diversas rutas urbanas entendidas siempre como áreas históricas urbanas.

De cada una de las áreas seleccionadas se analizará tres tipos de redes que, aunque están interrelacionadas, pueden estudiarse por separado:

- Red de Espacios Edificados

- Red de Espacios Libres

- Red de Infraestructuras

Cada una de estas redes será susceptible de seis tipos de análisis diferentes, entendiéndose siempre como enfoques desde los que comprender las mismas:

- Análisis Físico-Formal

- Análisis Funcional

- Análisis Económico

- Análisis Administrativo-Legal

- Análisis Medioambiental

- Análisis Histórico

De la interrelación de estos análisis se derivará el entendimiento de un Análisis Cultural de nuestro Patrimonio Urbano.

Por último cada uno de estos análisis deberá contemplar sus correspondientes Indicadores que intentarán recoger los valores, los riesgos y los recursos de cada una de las áreas estudiadas.

El desarrollo de este esquema tendrá que incidir en el nivel de profundización y de definición de cada grupo de indicadores, así como de la posibilidad de contemplar un módulo relacional de Conservación.
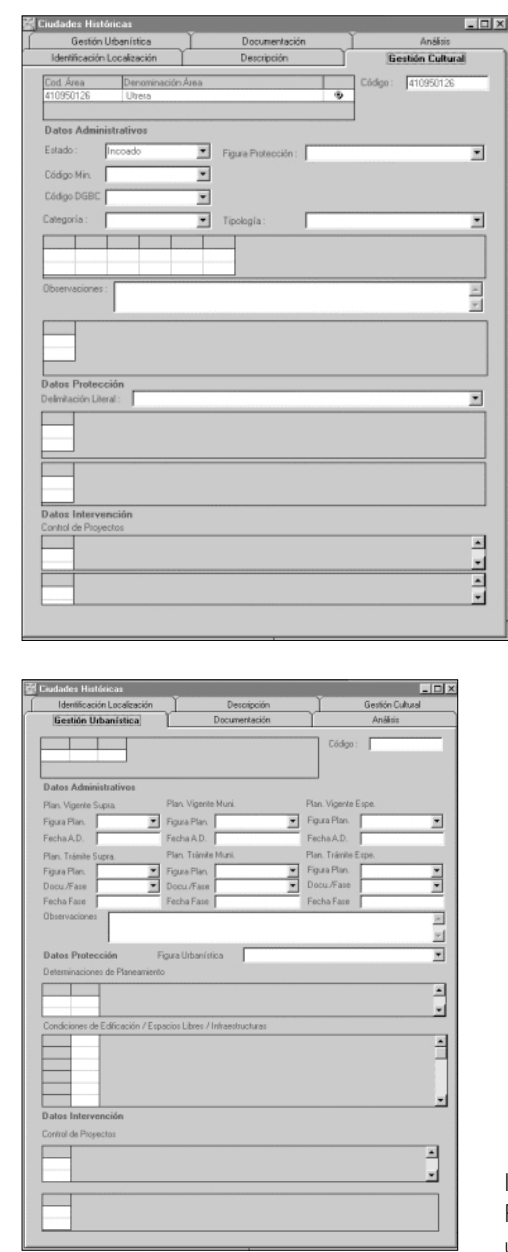

Imagen 6.

Gestión cultural.

Imagen 7.

Pantalla de gestión

urbanística.

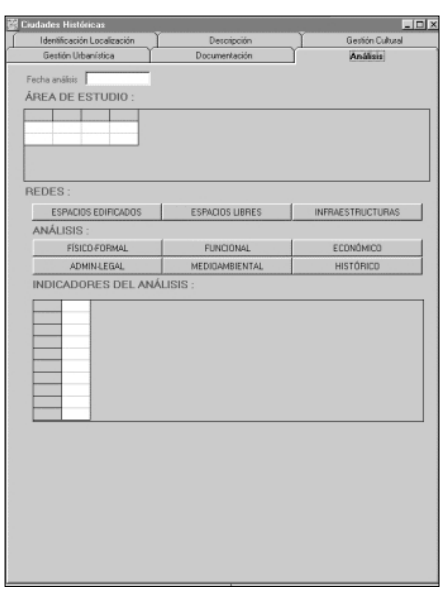

Imagen 8.

Pantalla de análisis.

Para terminar, decir que aprovechando este trabajo y la publicación del Cdrom de "Inmuebles protegidos en las capitales andaluzas", se ha iniciado una campaña de actualización de los datos, toda vez que se dispone de los últimos planeamientos urbanísticos generados para la ordenación y planificación del territorio andaluz.

Este trabajo se ha desarrollado por el área de patrimonio arquitectónico y urbano del centro de documentación del IAPH, coordinado por Mariano Pérez Humanes y participando en los criterios documentales y la recopilación de información Carlos Tapia Martín y Salud Soro Cañas. 